\title{
Good Financial Grant Practice (GFGP): A Tool for Developing \& \\ Demonstrating Institutional Financial \& Grant Management Capacity in
}

\section{Global Health}

Authors:

Harry J. Harste, Oxford Big Data Institute, University of Oxford, Oxford, United Kingdom;

Wellcome Genome Campus, Hinxton, United Kingdom

Genevieve Kiff, Oxford University Clinical Research Unit, Hanoi, Vietnam; African Academy of

Sciences, Nairobi, Kenya

Iruka N. Okeke, Global Health Research Unit for the Genomic Surveillance of Antimicrobial

Resistance, Department of Pharmaceutical Microbiology, Faculty of Pharmacy, University of Ibadan, Oyo State, Nigeria

Akindele O. Adebiyi, College Research and Innovation Management, College of Medicine, University of Ibadan, Nigeria

K. L. Ravikumar, Central Research Laboratory, Kempegowda Institute of Medical Sciences, Bengaluru, India

Geetha Nagaraj, Central Research Laboratory, Kempegowda Institute of Medical Sciences, Bengaluru, India

Jolaade J. Ajiboye, Department of Pharmaceutical Microbiology, University of Ibadan, Ibadan, Nigeria

Erik C. D. Osma Castro, Agrosavia (Corporación Colombiana de Investigación Agropecuaria), Bogotá, Colombia

Elmer Herrera, Antimicrobial Resistance Surveillance Reference Laboratory, Research Institute of Tropical Medicine, Manila, Philippines

David M. Aanensen, Oxford Big Data Institute, University of Oxford, Oxford, United Kingdom; Wellcome Genome Campus, Hinxton, United Kingdom The NIHR Global Health Research Unit on Genomic Surveillance of Antimicrobial Resistance ${ }^{\text {a }}$ 
${ }^{a}$ Members of the NIHR Global Health Research Unit on Genomic Surveillance of Antimicrobial Resistance are listed in the Acknowledgments.

Keywords: GFGP, Good Financial Grant Practice, research funding, grant management, governance

Running title: GFGP in global health

Corresponding author: Harry J. Harste, Oxford Big Data Institute, University of Oxford, Oxford, United Kingdom; Wellcome Genome Campus, Hinxton, United Kingdom. harry.harste@cgps.group. Alternate corresponding author: Genevieve Kiff, Oxford University Clinical Research Unit, Hanoi, Vietnam; African Academy of Sciences, Nairobi, Kenya. genny.kiff@tritoma.co.uk.

40-word summary: We describe our experiences of implementing Good Financial Grant Practice (GFGP) across four diverse research institutions in India, Nigeria, Colombia, and the Philippines. GFGP can be used to address some of the challenges of the current research funding model. 


\begin{abstract}
The administration and governance of grant funding across global health organizations presents enormous challenges. Meeting these challenges is crucial to ensuring that funds are used in the most effective way to improve health outcomes, in line with the United Nations' Sustainable Development Goal (SDG) 3, "Ensure healthy lives and promote well-being for all at all ages." The Good Financial Grant Practice (GFGP) Standard (ARS 1651) is the world's first - and currently only - international standard in the financial governance and management of grant funding. Through consensus building and global harmonization between both low and middle-income, and high-income country players, the GFGP Standard has achieved a levelling impact: GFGP applies equally to, and can be implemented by, all types of organization, regardless of location, size, or whether they predominantly give or receive funding.

GFGP can be used as a tool for addressing some of the challenges of the current funding model. We describe our experiences and lessons learned from implementing GFGP across four diverse research institutions in India, Nigeria, Colombia, and the Philippines, as part of our UK National Institute for Health Research Global Health Research Unit (GHRU) on Genomic Surveillance of Antimicrobial Resistance.
\end{abstract}




\section{ABBREVIATIONS \\ GFGP - Good Financial Grant Practice}

\section{FUNDING}

This work was supported by Official Development Assistance (ODA) funding from the National Institute of Health Research [grant number 16_136_111]. INO is supported by an African Research Leader Award from the UK Medical Research Council (MRC) and the UK Department for International Development (DFID) under the MRC/DFID Concordat agreement that is also part of the EDCTP2 programme supported by the European Union.

This research was commissioned by the National Institute of Health Research using Official Development Assistance (ODA) funding. The views expressed in this publication are those of the authors and not necessarily those of the NHS, the National Institute for Health Research or the Department of Health.

\section{CONFLICT OF INTEREST}

Dr. Okeke is a member of the Bill \& Melinda Gates Foundation (BMGF) Surveillance Advisors Group (SAG) (from 2019) and received grants and non-financial support from International Vaccine Institute. GK is a Senior Advisor to the African Academy of Sciences. All authors have submitted the ICMJE Form for Disclosure of Potential Conflicts of Interest. 


\section{Main manuscript}

\section{INTRODUCTION}

Unprecedented funds are being pledged and used to support public health research and services globally. In 2004, global health funding was around \$14 billion, and this increased rapidly, largely due to initiatives such as the Bill \& Melinda Gates Foundation and the US Government's AIDS initiative [1, 2]. In 2015, development assistance for health (DAH) funding alone accounted for around $\$ 19$ billion. Particular importance was placed on the 31 low-income countries where external sources of funding accounted for $33 \%$ of total health spending on average in 2015- with this proportion increasing over time in absolute terms [3].

The administration and governance of funds across a wide geographical range and number of global health organizations involves significant challenges. Meeting these challenges is crucial to ensuring funds are used most effectively to meet their intended aim of improving health outcomes, in line with the United Nations' Sustainable Development Goal (SDG) 3, "Ensure healthy lives and promote wellbeing for all at all ages" [4].

Developing institutional capacity to receive and administer funding at grantee organizations is a key component in ensuring that institutional structures are effective at managing large scientific grants to achieve intended research aims [5]. Similarly, the role of effective governance on health outcomes is crucial. Research has shown that poor governance and corruption contribute more to antibiotic resistance rates than antibiotic usage volumes, demonstrating the importance of governance in supporting effective health outcomes [6].

This paper describes the work undertaken as part of the UK National Institute for Health Research (NIHR) Global Health Research Unit (GHRU) on Genomic Surveillance of Antimicrobial Resistance in implementing the Good Financial Grant Practice (GFGP) Standard as a method of addressing some of the challenges of the funding model. 


\section{CHALLENGES \& DEVELOPMENTS IN THE FUNDING MODEL}

\section{Inadequate Institutional Capacity Support}

Despite the unprecedented flow of global health funding into low and middle-income countries (LMICs), there are often significant gaps in physical infrastructure, human capital, governance, and management structures (including procurement systems) in LMIC research institutes [5, 7-10]. These gaps include a lack of institutional financial and grant management capacity, creating major challenges around the administration of grant funding.

A lack of funding to support institutional capacity can create a vicious cycle, in which LMIC organizations are starved of support for indirect costs (also referred to as "overheads" - expenses that are not directly attributable to a specific project or grant, such as utilities and administrative staff costs) $[5,11]$. Continual, inadequate indirect cost support from grantors means grantees may increasingly struggle to function effectively, but they are often reluctant to ask for more support in case the request is detrimental to future funding applications and collaborations. This can create a perpetuating cycle of indirect cost underfunding [11-13]. Irrespective of how well a particular project is designed and implemented, if the organizational capacity to effectively manage its financial resources is not in place, there is likely to be a significant impact on the delivery and sustainability of the project [14].

However, adequately funding indirect costs alone is not always enough to develop institutional financial and grant management capacity. Capacity building support, for example by establishing and training a successful grants administration office, is sometimes also required to enable institutions to effectively use indirect cost funding, with increasing awareness that this support is a requisite component for managing grant funding [15]. For example, in 2018, the NIHR announced supplementary Financial Assurance Funding (FAF) to their Global Health Research awards for specific financial capacity-building activities with LMIC partners [16]. The NIHR has developed this approach further by allowing these costs to be included within direct program costs for their awards commencing in 2021 [17]. 


\section{Ineffective Governance}

Poor governance and corruption are fundamental challenges for the administration of grant funding, and they represent a significant barrier to universal health coverage, by preventing funding from reaching intended recipients and thereby directly impacting research projects and SDG 3. Estimating the scale of this problem is challenging, but it constitutes a significant issue in global health and beyond $[18,19]$. In addition, corruption has been shown to be a key socio-economic factor in explaining antibiotic resistance rates [6].

Effective governance within research institutions is crucial to ensure effective decision making, at a programmatic level and more widely, and to improve both accountability and trust within the institution and across its personnel. The development of effective governance through the development of institutional capacity is also an important component in the fight against corruption [18].

\section{Ineffective and Inefficient Due Diligence}

The current process for granting and receiving global health funding involves grantors, often highincome country (HIC)-based funders, asking multiple disparate due diligence questions of grantees (due diligence is an organizational review that is completed before entering into an agreement with that organization). There is often a lack of clarity and understanding of what is being asked for and what represents best practice, with due diligence requirements usually differing between funding agencies and institutions. There is also a power imbalance in this relationship, where grantors are requesting information before either programmatic activity begins or funds are transferred, putting onerous requirements on grantees in terms of staff time and cost of compliance. For example, in 2018 and 2019, one Nigerian university had more than 20 due diligence requests from 16 different global funders, including UNICEF, the African Academy of Sciences, the International Development Research Centre, UK National Institute of Health Research (NIHR), and UK Research and Innovation Global Challenges Research Fund. 
There is a clear need for the adoption of a standardized and more equitable approach to due diligence, through which LMICs and HICs contribute to the development of a due diligence framework that works for and is better understood by both grantors and grantees. This will improve the identification of key risks and reduce the duplication of effort, administrative burden, and costs for all players [20]. Various existing initiatives attempt to standardize an approach to due diligence. However, their scope is generally limited to specific sectors or countries, and they do not represent a globally harmonized approach [21].

\section{The Good Financial Grant Practice (GFGP) Standard}

The Good Financial Grant Practice (GFGP) Standard (ARS 1651) is the world's first - and currently only - public international quality standard in the financial governance and management of grant funding [22]. The GFGP Standard represents a paradigm shift in approach, using consensus building and global harmonization between LMIC and HIC players.

The standard was developed by the African Academy of Sciences (AAS) in Nairobi, Kenya in collaboration with global grantors and grantees from 22 countries across Eastern, Western, and Southern Africa. This involved some of the world's largest public and private sector funders, including those that provided funding - Wellcome, UK Research and Innovation, UK Department of Health \& Social Care, the IKEA Foundation, the European \& Developing Countries Clinical Trials Partnership (EDCTP) - and other collaborators: the African Union, UK Foreign, Commonwealth and Development Office (formerly DfID), the African Organisation for Standardisation and the New Partnership for Africa's Development and Coordinating Agency (NEPAD) [22].

The initiative was developed through a series of interactive workshops, with active participation from LMIC grantees and global grantors. GFGP creates a standard that is uniquely designed when compared to other standards or due diligence tools. The engagement of grantees and grantors from a wide range of countries and risk profiles in the design and development of GFGP has enabled the standard to effectively cover the key financial and grant management risks that affect organizations and directly impact programmatic delivery. 
The GFGP Standard is made up of four practice areas: Financial Management, Human Resources, Procurement, and Governance. Adherence follows a series of steps.

Firstly, organizations self-assess against one of the scheme's four tiers (bronze, silver, gold, and platinum); each tier is applicable to different types of organization (Figure 1). The identification of an appropriate tier is a crucial step in the process, as the tiers do not represent an incremental continuum but are designed for organizations of differing sizes and complexities. Self-assessment involves completing a pre-certification assessment through an online portal, where between 70 (bronze) and 300 (platinum) clauses are completed, and supporting procedural (bronze), process (silver), and policy (gold/platinum) documentation is uploaded. An organization can self-assess against GFGP at an institutional or departmental level. This decision is based on how the organization administers its grant funding, either by central administration function or by self-contained departmental administration function.

Secondly, once organizations have self-assessed, they can request to be certified (audited) against the GFGP Standard by a licensed auditor (certifying body (CB)). CBs will review the adequacy of the procedures, processes, and policies in addressing key organizational risks, and the practical implementation of the documents to address those risks (over at least the previous three months). If the $\mathrm{CB}$ assesses the organization as fully compliant, they will issue a certificate of compliance along with an accompanying audit report detailing findings. This process is supported by an online portal, where grantees can share their certificates and report findings with other grantors. The GFGP Standard, online portal, certification scheme, and CBs are collectively known as the Global Grant Community (GGC) [22].

\section{Developing Institutional Capacity}

To avoid accreditation disadvantaging low-resource settings, requirements need to be transparent and ordered, making it possible for institutions that have gaps in capacity to develop a roadmap to meeting requirements [23]. The GFGP Standard provides a clear framework for improving institutional financial and grant management capacity. 
When completing the GGC portal questionnaire, an organization can identify and track gaps in their current compliance. If this is the case, an implementation plan towards full GFGP compliance should be designed and undertaken. This is likely to include a series of recommendations around designing new documentation or refining existing documentation, and then embedding this in organizational practice. Organizations may look to engage an external GFGP implementation consultant or employ a financial and grant management specialist to identify gaps and design effective implementation plans. However, this may be a challenge in resource-constrained settings, where funders are unable or unwilling to provide funding. As the number of GFGP-certified organizations increases, there appears to be scope for cross-institutional mentoring in the initiation phase of the implementation process. Once an organization is confident that their documentation is adequate and has been practically implemented, a CB can be engaged to undertake the GFGP certification (audit). Certification gives the organization international recognition and gives funders and key stakeholders confidence in the effectiveness of institutional financial and grant management.

\section{Enhancing Institutional Governance}

There is a specific focus in the GFGP Standard on improving governance and therefore trust and accountability within organizations. In the development of the GFGP Standard, the importance of effective governance was recognized in the creation of a separate section on governance (section 8) containing four sub-sections (General governance, Audit, Grant compliance, and Risk management). For example, within the risk management sub-section of the standard (section 8.4), there is a focus on the mechanisms that organizations put in place to enable individuals to confidentially report incidents of possible fraud, corruption, and bribery. The CB will check to see whether the procedural steps around disclosing fraud, bribery, and corruption have been followed and can be demonstrated. In this example, any significant issues identified by the CB around governance would need to be addressed by the organization before the CB issues a certificate of GFGP compliance.

\section{Effective and Efficient Due Diligence}


Through consensus building of grantors and grantees from HICs and LMICs during the development process, the GFGP Standard has a levelling impact, which is significant in the due diligence process, when grantors request that grantees comply with certain requirements. This levelling impact is also created by a "one standard" approach, whereby GFGP applies equally to, and can be implemented by, all types of organization (Figure 1).

Organizations can use GFGP in both pre and post-award due diligence by requesting that another organization complete a self-assessment questionnaire on the GGC online portal against one of the standard's tiers. The requesting organization can then review the self-assessment responses on the GGC portal and follow up with any supplementary questions.

GFGP certification simplifies the due diligence process significantly. The GFGP certificate and accompanying report become evidence of compliance across the four practice areas, providing the assurance the grantor needs that the grantee organization is compliant to an international quality standard in grant management. The grantor may ask supplementary assurance questions. To support grantors to identify and ask supplementary questions, the GGC has also designed a set of non-GFGP assurance questions around Safeguarding, Programmatic delivery, Legal \& Compliance, and Health \& Safety [22].

Although there may be a significant initial investment of time and resources to achieve full GFGP certification, the benefits of simplified due diligence are considerable, with less time and money spent on financial and grant compliance and more resources available to support programmatic delivery.

\section{IMPLEMENTING GFGP IN GLOBAL HEALTH ORGANIZATIONS}

As part of the NIHR Global Health Research Unit (GHRU) on Genomic Surveillance of Antimicrobial Resistance, we have implemented the GFGP Standard with our four partners: a privatepublic non-profit research organization in Colombia (AGROSAVIA), a medical college in India (Central Research Laboratory, Kempegowda Institute of Medical Sciences (CRL KIMS)), a large 
public university in Nigeria (University of Ibadan (UI)), and a public hospital in the Philippines (Research Institute of Tropical Medicine (RITM)).

GFGP was principally implemented as a method of building and demonstrating sustainable financial and grant management capacity to complement the scientific capacity that has been developed through Whole Genome Sequencing (WGS) implementation [24, 25]. WGS is becoming the method of choice for applications including pathogen surveillance, biomarker discovery, and host response to disease, which have to be implemented in-situ. WGS approaches provide the opportunity to flexibly add on new capacities as well as to leapfrog earlier roadblocks to science and health advancement in LMICs [26-29]. In contrast to many other global health endeavors that focus heavily on staff and patients, implementing WGS additionally entails large purchase contracts, agile procurement systems, and predominantly high-level staffing. For these reasons, it is especially vulnerable to gaps in GFGP compliance, particularly across human resources and procurement.

In 2019, we worked with the four institutes to complete the GFGP self-assessment questionnaire on the GGC portal. Diagnostic assessment visits were then performed at each partner site, with the Nigeria assessment visit performed in partnership with Humentum [30]. These visits focused on assessing current levels of GFGP compliance and providing organizational implementation plans to reach full GFGP compliance.

The GFGP implementation lead worked remotely in collaboration with partner GFGP leads, to implement the plans to reach full GFGP compliance. Each partner is currently at a different stage of implementation.

AGROSAVIA has methodically implemented all the gold tier recommendations made following a diagnostic assessment visit in 2019 and is currently completing the certification process. The 2019 assessment visit estimated AGROSAVIA's overall compliance with the GFGP gold tier at around 80\%. Although AGROSAVIA had advanced systems and practices in place across all the GFGP practice areas, international grant funding was a relatively new funding stream for the organization, and therefore the development and formalization of the international elements of the standard, such as procedures and policies on exchange rates, indirect costs, and recording time/effort on grants, were critical to enable AGROSAVIA to fully comply with the GFGP gold tier requirements. 
RITM has established a GFGP committee but has yet to implement the bronze tier recommendations. This is largely due to the current challenges faced by RITM in being the principal COVID-19 testing site in the Philippines.

UI has also established a GFGP committee to address the bronze tier recommendations; however, this will require a transformational change within UI that may take a number of years to implement. Reaching GFGP certification is likely to be a challenging process for UI, but given the significant number of funders this public university works with and the large number of principal investigators served by the grant management platforms, and therefore the volume of due diligence requests and funding administered, certification is likely to have significant organizational benefit to the university. We intend to continue to work in partnership beyond the life of this project to address these issues. CRL KIMS is now fully GFGP-certified, as outlined in the following case study.

\section{Case Study: The World's First GFGP Certification}

CRL KIMS is a small research laboratory with a self-contained administration function. It has internationally certified (International Standards Organization (ISO) 1589:2012) research practices and facilities, but the level of grant management practices was not consistent. Prior to 2019, some financial and operational controls were in place, but these were not consistently applied or documented. A diagnostic assessment visit performed in 2019 identified overall compliance with the GFGP Standard at bronze tier of around $65 \%$.

Six steps were taken in implementing GFGP at CRL KIMS, from the identification of gaps in GFGP compliance through to successful GFGP certification (Figure 2). As a partnership, we drafted a customized manual of standard operating procedures (SOPs), using a similar format to CRL KIMS' laboratory (ISO) documentation. We created 21 individual SOPs, ensuring that the GFGP bronze tier requirements were met and that the procedures satisfied CRL KIMS' organizational needs. A governance committee was also constituted to ensure that CRL KIMS had adequate structures for effective governance in line with the GFGP Standard.

Once these procedures were finalized, they were translated into the local language, Kannada, to ensure effective communication among all team members who had different levels of ability in the 
English language. The translation process was critical for ensuring that all members of the team were comfortable with the detailed procedures.

The project then entered the practical implementation phase, focused on embedding the procedures and demonstrating at least three months of implementation to the CB (BDO LLP) [31]. This work culminated in the production of a detailed electronic certification file for the $\mathrm{CB}$ for remote delivery (driven by COVID-19 restrictions).

CRL KIMS was GFGP certified on June 15, 2020, with two minor non-conformities (noncompliances) noted in the audit findings report.

Certification has brought multiple benefits to CRL KIMS by providing a best-practice structure to the administration function, and developing staff knowledge and understanding of financial and grant management. Since certification, CRL KIMS' organizational credibility has been enhanced, and this has facilitated and expedited the formalization of relationships with external partners and funders. For example, a multinational vaccine company and an Indian private diagnostics company have recently formalized partnerships with CRL KIMS. Obtaining GFGP certification played a major role in reducing the time taken to formalize these agreements and release funds, by $40-50 \%$ (from between 10 to 12 weeks to around 6 weeks), when compared to similar agreements made prior to becoming GFGP certified.

There were a number of useful practical lessons learned from implementing GFGP at CRL KIMS and across our GHRU partners (Figure 3).

\section{Refinements to the GFGP Standard}

While the benefits of implementing GFGP can be considerable for institutions, our practical implementation has highlighted some areas that require refinement and enhancement. Firstly, some of the GFGP questions appear repetitive. For example, there is some crossover in bronze (procedural) and silver (process) tier questions. This contributes to a doubling of the number of questions between bronze and silver, from around 70 to 140 , but not a doubling of required effort to comply with the standard. Therefore, a review of bronze and silver requirements would provide greater distinction between the two tiers and remove any repetition. This would also ensure that the 
silver tier better represents more complex organizations, such as research organizations managing large grant awards, while enabling smaller organizations, such as community-based organizations, to comply with a streamlined bronze tier.

Secondly, although compliance with the GFGP Standard will have an overall positive impact on programmatic delivery for institutions, such as by ensuring appropriate human resource practices to support high-level staffing requirements, there are some areas of the standard where amendments would enhance programmatic delivery. For example, while the procurement section of the standard requires detailed processes and policies to be implemented, the standard doesn't specifically refer to the need for timely procurement. Inclusion of a clause to this effect would strengthen the standard by addressing the operational risk of sluggish implementation of the procurement practices, and the subsequent potential impact on programmatic delivery.

\section{THE FUTURE OF GFGP}

It is intended that the GFGP Standard will become an ISO standard. The Kenyan Bureau of Standards (on behalf of the African Organisation for Standardisation) has submitted GFGP to ISO as a new work item, and it has been accepted. Progressing to ISO is likely to represent a positive step in terms of driving global uptake of GFGP. However, there are a number of factors that need to be considered, and challenges to overcome, before this can become a reality.

There are currently around 420 organizations registered on the GGC portal. As this is a new standard, GFGP has yet to reach engagement by a critical mass of organizations or establish a position of longterm sustainability. To reach this point, GFGP requires an increasing number of funders to endorse or advise implementation of the standard and make GFGP-related costs allowable on their grants. This will drive uptake by organizations in the Global North and South, and across global health and more widely. The implementation of GFGP by organizations in the Global North is an important development to ensure that GFGP is a truly equitable initiative, regardless of geographical location or organizational type. 


\section{CONCLUSION}

The equitable development of GFGP has created a powerful new standard that addresses challenges within the global health funding model. In our experience of implementing the GFGP Standard, it has proved an effective tool for identifying and addressing key organizational risks, and for developing institutional financial and grant management capacity across global health partners in Africa, Asia, and Latin America. To fully assess the efficacy of the GFGP Standard, further work is required in implementing the standard more widely, and in analyzing the qualitative and quantitative impact of GFGP certification on institutions.

The next steps for GFGP are important ones, but GFGP's innovative design means that it is well placed to have a long-term future, with the potential to transform the way we give and receive global health funding. 


\section{ACKNOWLEDGMENTS}

Members of the NIHR Global Health Research Unit on Genomic Surveillance of Antimicrobial

Resistance: Khalil Abudahab, Monica Abrudan, Silvia Argimón, Mihir Kekre, Dawn Muddyman, Ben Taylor, Anthony Underwood, Nicole Wheeler, and Sophia David of the Centre for Genomic Pathogen Surveillance, Big Data Institute, University of Oxford, Old Road Campus, Oxford, United Kingdom and Wellcome Genome Campus, Hinxton, UK; Pilar Donado-Godoy, Johan Fabian Bernal, Alejandra Arevalo, and Maria Fernanda Valencia of the Colombian Integrated Program for Antimicrobial Resistance Surveillance - Coipars, CI Tibaitatá, Corporación Colombiana de Investigación Agropecuaria (AGROSAVIA), Tibaitatá - Mosquera, Cundinamarca, Colombia; Varun Shamanna, Vandana Govindan, Akshata Prabhu, D. Sravani, M. R. Shincy, Steffimole Rose, and Ravishankar K.N of the Central Research Laboratory, Kempegowda Institute of Medical Sciences, Bengaluru, India; Anderson O. Oaikhena, Ayorinde O. Afolayan, and Erkison Ewomazino Odih of the Department of Pharmaceutical Microbiology, Faculty of Pharmacy, University of Ibadan, Oyo State, Nigeria; Celia Carlos, Marietta L. Lagrada, Polle Krystle V. Macaranas, Agnettah M. Olorosa, and June M. Gayeta of the Antimicrobial Resistance Surveillance Reference Laboratory, Research Institute for Tropical Medicine, Muntinlupa, the Philippines; Ali Molloy, alimolloy.com; John Stelling, The Brigham and Women's Hospital; and Carolin Vegvari, Imperial College London.

The authors thank the following people for helpful discussions and contributions: Mrs Funmi Oladeji and Babasanmi Osinkolu from UI; Nethravathi Gowda, Rajitha Gowda, and Vandana Govindan from CRL KIMS; Marcela Vallejo Beltran, Natalia Andrea Mora Leon, and Edson Manuel Peña Moreno from AGROSAVIA; Sam Musoke, Juan Manuel Palacios, and Rudi Lewin from Humentum; Michael Kilpatrick, Edward Abira, Winfred Muasa, Vincent Nkundimana from the Global Grant Community; Gerald Bates from the International Institutions \& Donor Assurance team at BDO LLP. 


\section{REFERENCES}

1. Sridhar D, Batniji R. Misfinancing global health: a case for transparency in disbursements and decision making. Lancet. 2008; 372(9644): 1185-1191. doi:10.1016/S0140-6736(08)61485-3

2. Kates J, Morrison JS, Lief E. Global health funding: a glass half full? Lancet. 2006; 368(9531): 187-188. doi:10.1016/S0140-6736(06)69018-1

3. Xu K, Soucat A, Kutzin J, et al. New Perspectives on Global Health Spending for Universal Health Coverage. Geneva: World Health Organization, 2018. Available at: https://apps.who.int/iris/bitstream/handle/10665/259632/WHO-HIS-HGF-HFWorkingPaper-17.10eng.pdf. Accessed June 9, 2021.

4. United Nations Department of Economic and Social Affairs. Sustainable Development Goal 3: Ensure healthy lives and promote well-being for all at all ages. Available at: https://sdgs.un.org/goals/goal3. Accessed June 9, 2021.

5. Crane JT. Scrambling for Africa: AIDS, expertise, and the rise of American global health science. African Affairs, 2014; 114(454): 160-161. https://doi.org/10.1093/afraf/adu068

6. Collignon P, Athukorala PC, Senanayake S, Khan F. Antimicrobial resistance: the major contribution of poor governance and corruption to this growing problem. PLoS One. 2015; 10(3): e0116746. Published 2015 Mar 18. doi:10.1371/journal.pone.0116746

7. Chu KM, Jayaraman S, Kyamanywa P, Ntakiyiruta G. Building research capacity in Africa: equity and global health collaborations. PLoS Med. 2014; 11(3): e1001612. Published 2014 Mar 11. doi:10.1371/journal.pmed.1001612

8. Beran D, Byass P, Gbakima A, et al. Research capacity building-obligations for global health partners. Lancet Glob Health. 2017; 5(6): e567-e568. doi:10.1016/S2214-109X(17)30180-8 9. Bhutta Z. Practising just medicine in an unjust world. BMJ. 2003; 327(7422): 1000-1001. doi:10.1136/bmj.327.7422.1000

10. Feagins AR, Vuong J, Fernandez K, et al. The Strengthening of Laboratory Systems in the Meningitis Belt to Improve Meningitis Surveillance, 2008-2018: A Partners' Perspective. J Infect Dis. 2019; 220(220 Suppl 4): S175-S181. doi:10.1093/infdis/jiz337 
11. Eckhart-Queenan J, Etzel M, Prasad S. Pay-What-It-Takes Philanthropy. Stanford Social Innovation Review. 2016. Available at:

https://ssir.org/up_for_debate/article/pay_what_it_takes_philanthropy\#. Accessed June 9, 2021.

12. Goggins Gregory A, Howard D. The Nonprofit Starvation Cycle. Stanford Social Innovation Review, 2009. Available at: https://ssir.org/articles/entry/the_nonprofit_starvation_cycle. Accessed June 9, 2021.

13. Eckhart Queenan J, Allen J, Tuomala J. Stop Starving Scale: Unlocking the Potential of Global NGOs: Bridgespan, 2013. Available at: https://www.bridgespan.org/insights/library/pay-what-ittakes/unlocking-the-potential-of-global-ngos. Accessed June 9, 2021.

14. Asian Development Bank. Financial Due Diligence: A Methodology Note. January 2009. Available at: https://www.adb.org/documents/financial-due-diligence-methodology-note. Accessed June 9, 2021.

15. Crane JT, Andia Biraro I, Fouad TM, Boum Y 2nd, R Bangsberg D. The 'indirect costs' of underfunding foreign partners in global health research: A case study. Glob Public Health. 2018; 13(10): 1422-1429. doi:10.1080/17441692.2017.1372504

16. National Institute for Health Research. NIHR Global Health Research Units and Groups FAF Guidance. Available at: https://www.nihr.ac.uk/documents/nihr-global-health-research-units-andgroups-faf-guidance/22789. Accessed June 9, 2021.

17. National Institute for Health Research. Global Health Research Programmes - Core Guidance. Available at: https://www.nihr.ac.uk/documents/nihr-global-health-research-programmes-stage-2applications-core-guidance/24952. Accessed June 9, 2021.

18. García PJ. Corruption in global health: the open secret. Lancet. 2019; 394(10214): 2119-2124. doi:10.1016/S0140-6736(19)32527-9

19. May O. Fighting Fraud and Corruption in the Humanitarian and Global Development Sector. Routledge, 2019; 3-5.

20. Els C. Country-based pooled funds: The NGO perspective: Norwegian Refugee Council, 2019. Available at: https://www.nrc.no/resources/reports/country-based-pooled-funds-the-ngo-perspective/. Accessed June 9, 2021. 
21. ARMA UK. First Output From The 'Consolidated Approach To Assurance And Due Diligence' Project. Available at: https://arma.ac.uk/first-output-from-the-consolidated-approach-to-assuranceand-due-diligence-project/. Accessed June 9, 2021.

22. The Global Grant Community. Good Financial Grant Practice. Available at: https://www.globalgrantcommunity.com/. Accessed June 9, 2021.

23. Yao K, Maruta T, Luman ET, Nkengasong JN. The SLMTA programme: Transforming the laboratory landscape in developing countries. Afr J Lab Med. 2014; 3(3): 194. doi:10.4102/ajlm.v3i2.194

24. Kekre M, Arevalo SA, Valencia MF, et al. Integrating Scalable Genome Sequencing into Microbiology Laboratories for Routine AMR Surveillance. Clin Infect Dis 2021;73: In this supplement

25. Afolayan AO, Bernal JF, Gayeta JM, et al. Overcoming data bottlenecks in genomic pathogen surveillance. Clin Infect Dis 2021;73: In this supplement 26. Folarin OA, Happi AN, Happi CT. Empowering African genomics for infectious disease control. Genome Biol. 2014; 15(11): 515. Published 2014 Nov 7. doi:10.1186/s13059-014-0515-y

27. Okeke IN, Feasey N, Parkhill J, et al. Leapfrogging laboratories: the promise and pitfalls of hightech solutions for antimicrobial resistance surveillance in low-income settings. BMJ Global Health. 2020; 5: e003622

28. Tessema SK, Inzaule SC, Christoffels A, et al. Accelerating genomics-based surveillance for COVID-19 response in Africa. Lancet Microbe. 2020; 1(6): e227-e228. doi:10.1016/S2666$5247(20) 30117-8$

29. Argimón S, Masim MAL, Gayeta JM, et al. Integrating whole-genome sequencing within the National Antimicrobial Resistance Surveillance Program in the Philippines. Nat Commun. 2020; 11(1): 2719. Published 2020 Jun 1. doi:10.1038/s41467-020-16322-5

30. Humentum. Available at: https://www.humentum.org/. Accessed June 9, 2021.

31. BDO LLP. BDO United Kingdom. Available at: https://www.bdo.co.uk/en-gb/home. Accessed June 9, 2021. 


\section{FIGURE LEGENDS}

Figure 1: One Standard Accommodates All. The four levels of the GFGP Standard - Platinum, Gold, Silver, and Bronze - detailing the types of organization for which each level is intended. Adapted with permission from the original, by the Global Grant Community, African Academy of Sciences.

Figure 2: CRL KIMS' journey to certification. The six steps undertaken by CRL KIMS in India, from identification of gaps in compliance to full GFGP certification.

Figure 3: Practical tips for implementing GFGP. 


\section{FIGURES}

Figure 1

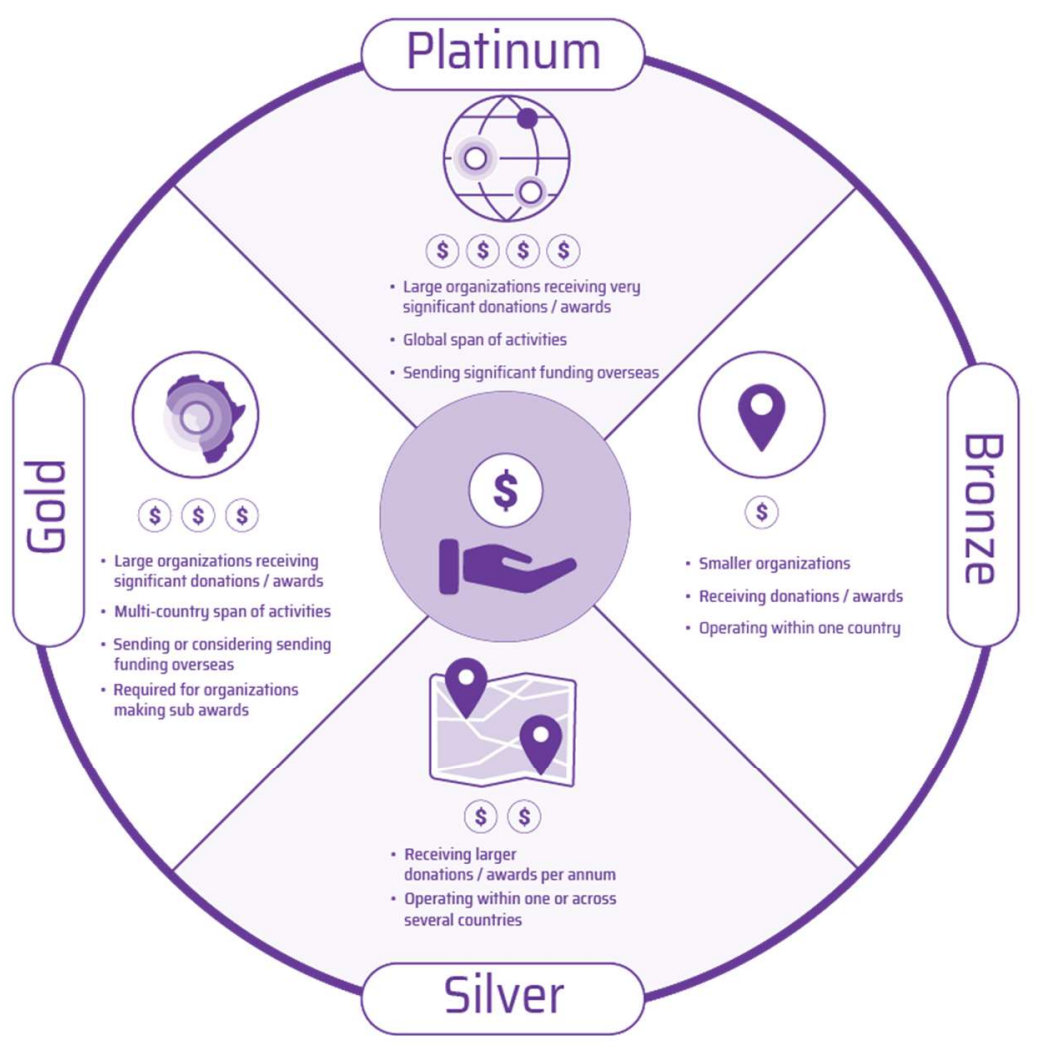

Figure 2

CRL KIMS journey towards GFGP certification

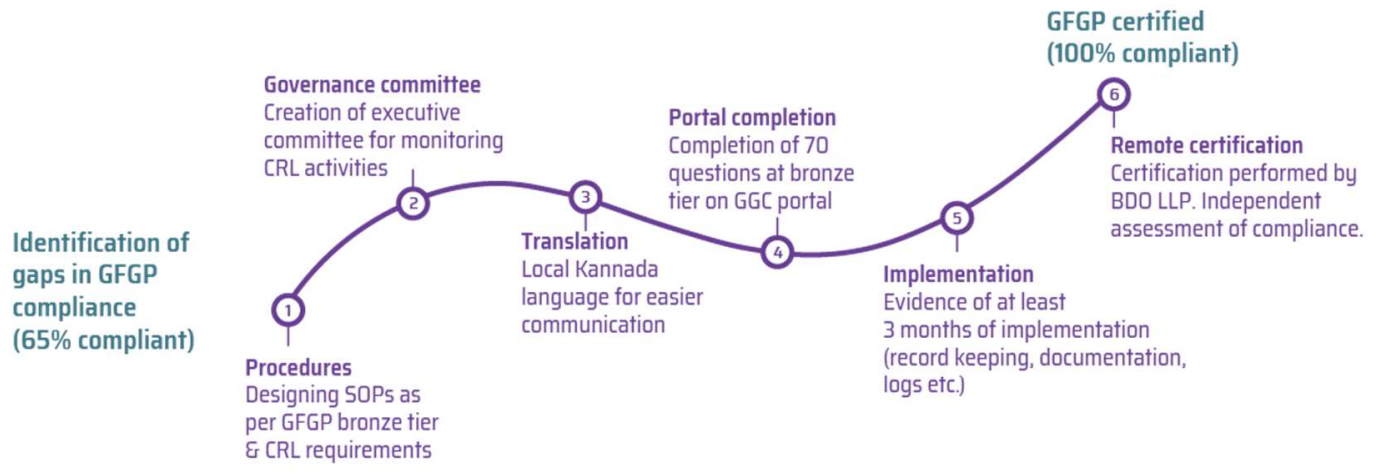


Figure 3

2 Determine the appropriate tier for your organization by assessing your organization's complexity, size, and geographical reach.

Get endorsement and agreement from senior members of staff from the start of the implementation process, as you may need this support to overcome areas of non-compliance.

Consider what documentation approach is best for your organization (i.e. do you need to create a manual of procedures, processes, and policies, or is it better to refine existing documentation?).

Appoint a GFGP project coordinator who is responsible for leading the implementation process and standardizing the self-assessment responses on the GGC portal.

Initially review all GFGP questionnaire questions to identify themes that run across multiple sections.

Break down the self-assessment questionnaire into sections, and tackle 1-2 at a time.

Involve multiple (relevant) members of staff in each GFGP section by discussing: each GFGP question;

the current compliance of documentation and practices; and what is required to address areas of non-compliance.

Be honest and self-critical when completing the self-assessment, as the certifier will be when they test your compliance. 